\title{
Effect of mobilizing agents on mycoremediation and impact on the indigenous microbiota
}

\author{
Maria A Giubilei, ${ }^{a}$ Vanessa Leonardi, ${ }^{\text {a Ermanno Federici, }}{ }^{\text {b }}$ Stefano Covino, ${ }^{\text {a }}$ \\ Vaclav Šašek, ${ }^{c}$ Cenek Novotny, ${ }^{c}$ Federico Federici, ${ }^{a}$ Alessandro D'Annibale ${ }^{a}$ \\ and Maurizio Petrucciolia*
}

\begin{abstract}
BACKGROUND: Mobilizing agents (MAs) have been suggested to improve the fungal degradation of polycyclic aromatic hydrocarbons (PAHs) in soil. Three different MAs (Tween 20, Tween 80 and soybean oil) were investigated for their ability to stimulate contaminant degradation by either Phlebia sp. DABAC 9 or Allescheriella sp. DABAC1 in a soil spiked with a mixture of PAHs.

RESULTS: Phlebia sp. and Allescheriella sp. markedly differed in their growth capabilities under non-sterile conditions and without MAs (3.0 versus $0.1 \mu \mathrm{g}$ ergosterol $\mathrm{g}^{-1}$ soil, respectively). However, soybean oil led to a 35 -fold increase of $A$ llescheriella sp. growth. Contaminant degradations by Phlebia sp. DABAC 9 and Allescheriella sp. DABAC 1 were best supported by soybean oil and Tween 20, respectively. Enumeration of cultivable bacteria and denaturing gradient gel electrophoresis (DGGE) analysis of PCR-amplified 16S rRNA showed that microbial density and biodiversity were positively affected by the mycoremediation especially with Allescheriella sp., the use of which led to an evident detoxification.
\end{abstract}

CONCLUSIONS: Allescheriella sp. DABAC 1 appears to be a promising strain in the remediation of PAH-contaminated soils. The different response of the two fungi to MAs addition confirms the stringent need for a preliminary lab-scale assessment of fungus/MA combinations prior to application.

(c) 2009 Society of Chemical Industry

Keywords: mycoremediation; polycyclic aromatic hydrocarbons; biodegradation; mobilizing agents; DGGE; microbial community structure

\section{INTRODUCTION}

Polycyclic aromatic hydrocarbons (PAHs) are ubiquitous contaminants in both terrestrial and aquatic ecosystems. ${ }^{1}$ Several PAH members have been included within the list of priority pollutants due to their toxic, mutagenic and carcinogenic effects. The concomitant low water solubility and bioavailability of these contaminants make them rather recalcitrant to microbial attack. ${ }^{2}$ Consequently, the biological degradation of PAHs has been shown to be promoted by additives able to mobilize them from the soil organic phase to the aqueous phase. ${ }^{3}$ Among PAH-degrading organisms, white-rot fungi (WRF) are particularly interesting due to their unspecific, radical-based degradation machinery, which operates mainly in the extracellular environment. ${ }^{4}$ Moreover, the filamentous growth enables these organisms to readily diffuse into soil and to actively penetrate within its micro-aggregates thus reaching contaminants and acting as dispersion vectors of autochthonous bacteria able to degrade pollutants. ${ }^{5}$

For the above reasons, the combined use of surfactants and WRF in soil remediation has received some attention, leading to largely variable results in terms of decontamination efficiency, ${ }^{6-8}$ impact on the indigenous microbiota ${ }^{9,10}$ and release of PAH-degrading enzymes. ${ }^{10,11}$ Most studies on this specific topic point out the importance of finding appropriate WRF mobilizing agent (MA) combinations. $7,12,13$ Another recurring point related to the use of MAs in soil remediation concerns their concentrations, which generally have to be well above their critical micelle concentrations as extensively reviewed by Haigh. ${ }^{14}$ This emphasizes the utmost importance of using cost-effective MAs, such as plant oils, as a valuable alternative to synthetic ones. ${ }^{12,15,16}$ Moreover, there is an ever increasing interest in the use of fungi isolated from historically contaminated sites in bioaugmentation due to their presumed

\footnotetext{
Correspondence to: Maurizio Petruccioli, Dipartimento di Agrobiologia \& Agrochimica, University of Tuscia, Via San Camillo De Lellis s.n.c., 01100 Viterbo, Italy.

E-mail:petrucci@unitus.it

a Department of Agrobiology and Agrochemistry, University of Tuscia, Viterbo, Italy

b Department of Cellular and Environmental Biology, University of Perugia, Perugia, Italy

c Experimental Mycology Laboratory, Institute of Microbiology, Academy of Sciences of Czech Republic, Prague, Czech Republic
} 